\title{
FAUNA DE VESPAS PARASITOIDES (INSECTA, HYMENOPTERA) COLETADAS EM UM FRAGMENTO DE CERRADO, JATAÍ, GOIÁS, BRASIL
}

\author{
A.B. Moraes $^{1 *}$, P. Perre ${ }^{2 * *}$, J.F. Sobczak ${ }^{3 * * *}$ \\ ${ }^{1}$ Universidade Estadual de Campinas, Departamento de Biologia Animal, CP 6109, CEP13083-970, Campinas, \\ SP, Brasil. E-mail: dri_morais@hotmail.com
}

\section{RESUMO}

Este é um estudo pioneiro envolvendo a fauna de parasitoides da região Sudoeste do Estado de Goiás, além de ser um dos poucos realizados em um fragmento de Cerrado. Em um período de 20 dias, foi realizado o levantamento dos himenópteros parasitoides utilizando três métodos de captura: Malaise, Möricke e varredura na vegetação. Foram encontrados parasitoides de sete superfamílias distribuídos em 27 famílias, sendo as mais abundantes Ichneumonidae e Ceraphronidae. Nenhum método de captura coletou todas as famílias encontradas, comprovando que o uso conjunto dos três métodos é o ideal para um estudo de levantamento da fauna de himenópteros parasitoides de uma região ou fragmento. As vespas parasitoides atuam como agentes de controle biológico e polinizadores, assim, ainda que pequeno (apenas $16 \mathrm{ha}$ ), o fragmento amostrado desempenha importantes funções ambientais, demonstrando a relevância de manter fragmentos de matas nativas próximos às plantações.

PALAVRAS-CHAVE: Hymenoptera, parasitoides, biodiversidade, Cerrado.

\section{ABSTRACT}

PARASITOID WASP FAUNA (INSECTA, HYMENOPTERA) COLLECTED IN A FRAGMENT OF CERRADO, JATAÍ, GOIÁS, BRAZIL. This is a pioneering study involving the fauna of parasitoids in the southwestern region of Goiás State, Brazil, besides being one of the few conducted in a fragment of Cerrado. In a period of 20 days, hymenopteran parasitoids were colleted using three methods of capture: Malaise, Möricke and sweep net in the vegetation. Parasitoids of 7 superfamilies were found, distributed in 27 families, the most abundant being Ichneumonidae and Ceraphronidae. No method of capturing collected all the families present, showing that the combined use of three of these methods is ideal for a study of fauna survey of hymenopteran parasitoids of a region or fragment. Parasitoid wasps act as biological agents and pollinators, thus, even if small (only $16 \mathrm{ha}$ ), the sample fragment performed important environmental functions and demonstrated the importance of maintaining native forest fragments near the crops/plantations.

KEY WORDS: Hymenoptera, parasitoid, biodiversity, cerrado.

O Cerrado é considerado o segundo maior bioma do Brasil em extensão, atrás somente da floresta Amazônica, representando cerca de 21\% do território nacional (KLINK; MACHADO, 2005). Seu clima é considerado estacional, com período chuvoso (outubro a março), um período seco, (abril a setembro), precipitação média anual de 1.500 mm e temperatura média entre 22 a $27^{\circ} \mathrm{C}$ (KLINK; MACHADO, 2005).
A fragmentação desse bioma teveiníciona década de 70 com a utilização da agricultura mecanizada (SOARES, 2005), sendo a responsável pela atual paisagem de vegetação remanescente em formato de ilhas desconectadas inseridas em uma matriz totalmente agrícola (ARRUdA, 2001). O Estado de Goiás, onde este estudo foi conduzido, é considerado o único estado brasileiro integralmente inserido no bioma Cerrado e, também, onde o desmatamento tem

${ }^{2}$ Universidade de São Paulo, Escola Superior de Agricultura "Luiz de Queiroz", Departamento de Entomologia e Acarologia, Piracicaba, SP, Brasil.

${ }^{3}$ Universidade Federal de São Carlos, Departamento de Ecologia e Biologia Evolutiva, São Carlos, SP, Brasil.

*Programa de Pós-graduação em Parasitologia, Depto. Biologia Animal, Unicamp.

**Programa de Pós-graduação em Entomologia, Depto. Entomologia e Acarologia, USP/ESALQ.

***Programa de Pós-graduação em Ecologia e Recursos Naturais, Depto. Ecologia e Biologia Evolutiva, UFSCar. 
sido bastante intenso. Estima-se que somente 35\% do Estado (aproximadamente, $122.805 \mathrm{~km}^{2}$ ) sejam ainda cobertos com formações savanicas e florestais remanescentes (SANO et al., 2008).

O Cerrado, considerado a savana tropical mais diversificada do mundo, possui uma biodiversidade altíssima (KLINK; MACHADO, 2005). A classe Insecta apareceem destaque, CAVALCANTI; Joly (2002) mostraram que $35 \%$ das abelhas, $13 \%$ das borboletas e $23 \%$ dos cupins registrados para a região Neotropical podem ser encontrados no Cerrado.

Os parasitoides, querepresentamaté $20 \%$ detodas as espécies de insetos presentes em um ecossistema (LASALLE; GAULD, 1993), possuem grande importância na regulação populacional de insetos (BORROR et al., 1992) e, por fazerem parte de um sistema tri-trófico, são considerados um grupo "bioindicador" da diversidade local. Apesar de sua importância (econômica e ecológica) e alta diversidade, pouco se conhece sobre a estrutura das comunidades de parasitoides nos trópicos (LASALLE, 1993).

O objetivo deste trabalho foi realizar o levantamento da fauna de himenópteros parasitoides em um pequeno fragmento de Cerrado.

As coletas foram realizadas em um fragmento localizado no distrito de Estância $\left(17^{\circ} 33^{`} S, 5^{\circ} 53^{\prime} \mathrm{W}\right)$ pertencente ao Município de Jataí, Goiás. Este fragmento, que constitui uma Reserva Legal, é formado por 16 ha de cerrado strict sensu (Fig. 1B), sem nenhuma conexão com outro fragmento e está inserido em uma matriz com predominância de monoculturas de soja, milho e áreas de pastagem (Fig. 1A).

Tabela 1 - Número de indivíduos e porcentagem das famílias de parasitoides coletadas com três diferentes métodos de captura em um fragmento de Cerrado, Jataí, Goiás.

\begin{tabular}{|c|c|c|c|c|c|c|c|}
\hline Superfamílias & $\begin{array}{c}\mathrm{N}^{\mathrm{o}} \text { de } \\
\text { indivíduos }\end{array}$ & Porcentagem & $\begin{array}{c}\quad \mathrm{N}^{o} \text { de } \\
\text { indivíduos }\end{array}$ & Porcentagem & $\begin{array}{c}\quad \mathrm{N}^{\mathrm{o}} \mathrm{de} \\
\text { indivíduos }\end{array}$ & Porcentagem & Porcentagem \\
\hline Famílias & Varredura & Varredura & Möericke & Möericke & Malaise & Malaise & Total \\
\hline \multicolumn{8}{|l|}{ Chalcidoidea } \\
\hline Agaonidae & 0 & 0 & 0 & 0 & 2 & 0,4 & 0,2 \\
\hline Aphelinidae & 2 & 1,1 & 0 & 0 & 9 & 1,6 & 1,4 \\
\hline Chalcididae & 1 & 1,4 & 0 & 0 & 5 & 0,9 & 0,7 \\
\hline Encyrtidae & 2 & 2,9 & 5 & 2,7 & 50 & 9,2 & 7,2 \\
\hline Eucharitidae & 1 & 1,4 & 0 & 0 & 3 & 0,5 & 0,5 \\
\hline Eulophidae & 4 & 5,8 & 0 & 0 & 22 & 4,1 & 3,3 \\
\hline Eupelmidae & 0 & 0 & 0 & 0 & 20 & 3,7 & 2,5 \\
\hline Eurytomidae & 0 & 0 & 0 & 0 & 5 & 0,9 & 0,6 \\
\hline Mymaridae & 0 & 0 & 1 & 0,5 & 14 & 2,6 & 1,9 \\
\hline Ormyridae & 0 & 0 & 0 & 0 & 3 & 0,5 & 0,4 \\
\hline Perilampidae & 0 & 0 & 0 & 0 & 6 & 1,1 & 0,7 \\
\hline Pteromalidae & 8 & 11,7 & 1 & 0,5 & 16 & 2,9 & 3,1 \\
\hline Signiphoridae & 0 & 0 & 0 & 0 & 7 & 1,2 & 0,9 \\
\hline Tanaostigmatidae & 0 & 0 & 1 & 0,5 & 8 & 1,5 & 1,1 \\
\hline Torymidae & 2 & 2,9 & 0 & 0 & 7 & 1,2 & 1,1 \\
\hline \multicolumn{8}{|l|}{ Ceraphronoidea } \\
\hline Ceraphronidae & 1 & 1,4 & 45 & 24,3 & 92 & 17 & 17,4 \\
\hline Megaspilidae & 0 & 0 & 3 & 1,6 & 7 & 1,2 & 0,1 \\
\hline \multicolumn{8}{|l|}{ Chrysidoidea } \\
\hline Bethylidae & 0 & 0 & 8 & 4,3 & 29 & 5,4 & 4,6 \\
\hline Chrysididae & 0 & 0 & 0 & 0 & 2 & 0,4 & 0,2 \\
\hline Dryinidae & 16 & 18,3 & 0 & 0 & 8 & 1,5 & 3 \\
\hline \multicolumn{8}{|l|}{ Cynipoidea } \\
\hline Figitidae & 0 & 0 & 1 & 0,5 & 1 & 0,2 & 0,2 \\
\hline Ibaliidae & 0 & 0 & 1 & 0,5 & 2 & 0,4 & 0,4 \\
\hline \multicolumn{8}{|l|}{ Ichneumonoidea } \\
\hline Braconidae & 2 & 2,9 & 12 & 6,5 & 17 & 3,1 & 3,9 \\
\hline Ichneumonidae & 25 & 36,7 & 69 & 54 & 80 & 14,8 & 21,9 \\
\hline \multicolumn{8}{|l|}{ Platygastroidea } \\
\hline Platygastridae & 4 & 5,8 & 21 & 11,3 & 70 & 12,9 & 11,9 \\
\hline \multicolumn{8}{|l|}{ Proctotrupoidea } \\
\hline Diapriidae & 1 & 1,4 & 15 & 8,1 & 56 & 10,3 & 9 \\
\hline Pelecinidae & 1 & 1,4 & 0 & 0 & 0 & 0 & 0,1 \\
\hline Total & 70 & 100 & 183 & 100 & 541 & 100 & 100 \\
\hline
\end{tabular}


Os espécimes foram coletados entre os dias 20 de maio e nove de junho de 2009, totalizando um período de coleta de 20 dias. Foram utilizados três métodos de coleta: armadilha Malaise (modelo Townes 1972), armadilha Möericke, e varredura com uso de uma rede entomológica na vegetação. Segundo Noyes (1989), o uso das armadilhas Malaise, Möricke e varredura na vegetação em conjunto, são eficientes para a amostragem rápida de vespas parasitoides.

As armadilhas Malaise constituíam em uma tenda de malha fina, branca na parte superior e preta nas laterais, com 2,10 m de cumprimento, com um frasco coletor contendo uma solução de Dietrich localizado no topo da armadilha. Foram instaladas duas armadilhas Malaise a $100 \mathrm{~m}$ da borda do fragmento, os quais permaneceram coletando durante período de 20 dias, sendo que as duas armadilhas constituíram uma única amostra no final das coletas.

A armadilha Möricke constituiu de uma bacia amarela de, aproximadamente, $30 \mathrm{~cm}$ de diâmetro por $10 \mathrm{~cm}$ de profundidade, contendo uma mistura de $300 \mathrm{~mL}$ de água, $2 \mathrm{~mL}$ de detergente e $2 \mathrm{~mL}$ de formol. Estas armadilhas foram colocadas a $3 \mathrm{~m}$ uma da outra ao longo de um transecto de $200 \mathrm{~m}$ dentro do fragmento. A cada 48 horas o seu conteúdo era removido e o material de interesse acondicionado em tubos plásticos contendo álcool $70 \%$.

A varredura na vegetação foi realizada com auxilio de uma rede entomológica de tecido de algodão com $50 \mathrm{~cm}$ de diâmetro e fixada a um cabo de madeira usado para manipular a rede contra a vegetação. A rede foi batida contra a vegetação dentro do fragmento em movimentos regulares por um total de 30 minutos cada coleta.

No laboratório, foram selecionados apenas os insetos pertencentes à ordem Hymenoptera da série Parasítica de todas as amostras. Este material foi identificado no nível de família com auxílio de chaves de identificação dos autores, HANSON; GAULD (2006) e Costa; BerTi-FilHo (2010).

Foram amostrados 794 exemplares de himenópteros parasitoides distribuídos em sete superfamílias e 27 famílias (Tabela 1). As famílias com maior predominância de indivíduos foram Ichneumonidae e Ceraphronidae, com 21,9\% e 17,4\% do total das amostras, respectivamente.

As armadilhas Malaise foram as que capturam o maior número de famílias e indivíduos, 26 e 541, respectivamente.Somentea família Pelecinidaenãofoi capturada por essa armadilha e as famílias Agaonidae, Eupelmidae, Eurytomidae, Ormyridae, Perilampidae, Signiphoridae, Tanaostigmatidae e Chrysididae somente foram capturadas por este método. A família que obteve o maior número de indivíduos coletados por essas armadilhas foi Ceraphronidae, com $17 \%$ do total amostrado. As armadilhas Möricke e varredura capturaram um número próximo de famílias, 13 e 14, respectivamente, apesar da grande diferença no número de indivíduos, 183 e 70 . O que pode ser justificado devido diferenças no esforço amostral de cada método. As armadilhas Mörickenão capturaramuma família exclusiva, já varredura na vegetaçãofoi oúnico métodoquecoletou indivíduos da família Pelecinidae. Em ambos os métodos, a família Ichneumonidae foi a mais predominante.
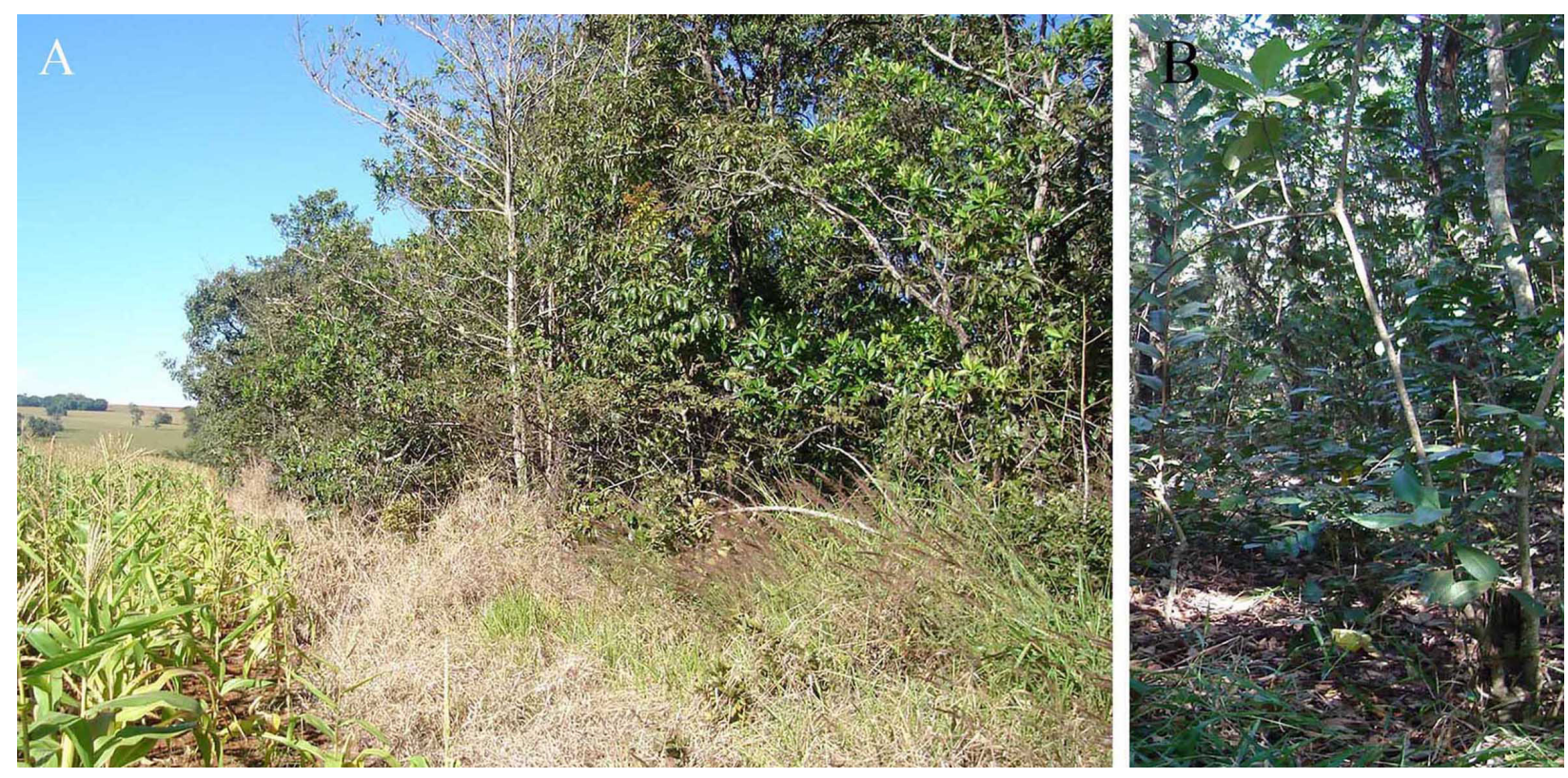

Fig. 1 - A) vista lateral da borda do fragmento amostrado, a esquerda uma plantação de milho e ao fundo pastagem; B) vista interna do fragmento. 
Algumas famílias somenteforam capturadas pela armadilha Malaise, o que indica que esses indivíduos possuem um habitat mais aéreo e usam o voo na procura de seus hospedeiros. Já a família Pelecinidae só foi capturada pelo método de varredura na vegetação, indicando que essa família deve habitar e utilizar o mesmo substrato que seus hospedeiros. Nenhum método de captura coletou todas as famílias encontradas, comprovando que o uso desses três métodos associados é o ideal para um estudo de levantamento da fauna dehimenópteros parasitoides de uma região ou fragmento.

Este é um estudo pioneiro envolvendo coleta de parasitoides na região Sudoeste do Estado de Goiás, além de ser um dos poucos realizados em fragmentos de Cerrado. Apesar das coletas terem sido realizadas em um curto espaço de tempo (20 dias), o número de exemplares e famílias coletados pode ser considerado bastante representativo, levando em consideração o tamanho reduzido do fragmento amostrado. Em um estudo similar, também realizado em um fragmento de Cerrado, porém com o tempo de coleta de 10 dias, MARCHIORI et al. (2007) coletaram apenas 135 indivíduos.

O número de famílias amostradas neste estudo corresponde a $30 \%$ das famílias de himenópteros conhecidas [90 famílias ao todo, HANSON; GAULD (2006)], o que indica que o Cerrado, mesmo sofrendo com o processo de fragmentação, ainda pode ser considerado um bioma com grande biodiversidade. Em relação às famílias com maior presença, este estudo apresentou resultados semelhantes aos encontrados por MARCHIORI et al., (2007), que coletaram em uma área de Cerrado e diferentes dos encontrados por Alencar et al. (2007), que amostraram em uma área de Mata Atlântica. Em um estudo sobre a estruturação da comunidade da família Ichneumonidae, SHAPIRO; PICKERING (2000) observaram que a umidade é um fator importante, o que explica resultados discrepantes em relação à presença dessa família em levantamentos feitos em áreas de Mata Atlântica (Azevedo et al., 2003), áreas agrícolas do Estado de São Paulo (SouzA et al., 2006) e florestas úmidas da Costa Rica (GASTON et al., 1996; Shapiro; Pickering, 2000).

Levando-se em consideração outros estudos que usaram os mesmos métodos empregados neste trabalho, observa-se que apesar do fragmento amostrado ser relativamente pequeno (apenas 16 ha), ainda é possível observar uma grande abundância de insetos parasitoides nesta área. Estes insetos desempenham uma importante função ambiental, atuando no controle biológico e, desta forma, auxiliando no combate a injúrias causadas por insetos pragas comuns em plantações. Esse estudo demonstra que pequenos fragmentos em áreas agrícolas são importantes para a manutenção da biodiversidade e, portanto, a sua preservação e manutenção em áreas agrícolas podem contribuir para a diminuição de insetos pragas.

\section{REFERÊNCIAS}

ALENCAR, I.D.C.C.; FRAGA, F.B.; TAVARES, M.T.; AZEVEDO, C.O. Perfil da fauna de vespas parasitoides (Insecta, Hymenptera) em áreas de Mata Atlântica do Parque Estadual da Pedra Azul, Domingos Martins, Espírito Santo, Brasil. Arquivos do Instituto Biológico, São Paulo, v.74, n.2, p.111-114, 2007.

ARRUDA, M.B. (Ed.) Ecossistemas brasileiros. Brasília: Ibama, 2001. 51p.

AZEVEDO, C.O.; CORRÊA, M.S.; GOBBI, F.T.; KAWADA, R.; LANES, G.O.; MOREIRA, A.R.; REDIGHIERI, E.S.; SANTOS, L.M.; WAICHERT, C. Perfil das famílias de vespas parasitóides (Hymenoptera) em uma área de Mata Atlântica da Estação Biológica de Santa Lúcia, Santa Tereza, ES, Brasil. Boletim Museu de Biologia Mello Leitão, v.16, p39-46, 2003.

BORROR, D.J.; TRIPLEHORN, C.A.; JOHNSON, N.F. An introduction to the study of insects. New York: Saunders College Publishing, 1992. 875p.

CAVALCANTI, R.; JOLY, C. The conservation of the Cerrados. In: OLIVEIRA, P.S.; MARQUIS, R.J. (Ed.). The Cerrado of Brazil. Ecology and Natural History of a Neotropical Savanna. New York: Columbia University Press, 2002. p.351-367.

COSTA, V.A.; BERTI-FILHO, E. Identificação das principais famílias de himenópteros parasitoides que ocorrem no Brasil. Campinas, 2010. 45p.

GASTON, K.J.; GAULD, I.D.; HANSON, P. The size and composition of the hymenopteran fauna of Costa Rica. Journal of Biogeography, v.23, p.105-113, 1996.

HANSON, P.E.; GAULD, I.D. (Ed.). Hymenoptera de la Región Neotropical. Memories of the American Entomological Institute, v.77. p.1-994, 2006.

KLINK, C.A.; MACHADO, R.B. Conservation of Brazilian Cerrado. Conservation Biology, v.19, p.707-713, 2005.

LASALLE, J.; GAULD, I.D. Hymenoptera: their diversity, and their impact on the diversity of other organisms. In: LASALLE, J.; GAULD, I.D (Ed.). Hymenoptera and biodiversity. Wallingford, UK: CAB International, 1993. p.1-26.

LASALLE, J. Parasitic hymenoptera, biological control and biodiversity. In: LASALLE, J.; GAULD, I.D. (Ed.). Hymenoptera and Biodiversity. Wallingford, UK: C.A.B. International, 1993. p.197-215.

MARCHIORI, C.H.; LUSSARI, M.A.; ROSA, D.C.; PENTEADO-DIAS, A.M. Parasitoid Hymenoptera 
colleted during the diurnal and nocturnal periods in Itumbiara, Goiás. Brazilian Jounal of Biology, v.67, n.3, p.581-582, 2007.

NOYES, H.S. The study of five methods of sampling Hymenoptera (Insecta) in a tropical rainforest, with special reference to the Parasitica. Journal of Natural History, v.23, n.3, p.285-298, 1989.

SANO, E.E.; DAMBRÓS, L.A.; OLIVEIRA, G.C.; BRITES, R.S. Padrões de cobertura de solos do Estado de Goiás. In: FERREIRA, L.G. A encruzilhada socioambiental - biodiversidade, economia e sustentabilidade no cerrado. Goiânia: UFG, 2008. p.91-106.

SHAPIRO, B.A.; PICKERING, J. Rainfall and parasitic wasp (Hymenoptera, Ichneumonoidea) activity in succesional forest stages at Barro Colorado Nature Monument,
Panama, and La Selva Biological Station, Costa Rica. Agricultural and Forest Entomology, v.2, p.39-47, 2000.

SOARES, J.L.N. Modelo agroecológico de desenvolvimento rural para os projetos de reforma agrária no Cerrado. Belém: 2005, 11p. (Documento Técnico). Disponível em: <http://www.icarrd.org/fr/icard_doc_down/others_ nead2.pdf $>$. Acesso em: 22 mar. 2011.

SOUSA, L.; BRAGA, S.M.P.; CAMPOS, M.J.O. Himenópeteros parasitóides (Insecta, Hymenoptera) em área agrícola de Rio Claro, SP, Brasil. Arquivos do Instituto Biológico, São Paulo, v.73, n.4, p.465-469, 2006.

Recebido em 3/4/11

Aceito em 18/5/12 